\title{
Overlapping Cell Nuclei Segmentation Using a Spatially Adaptive Active Physical Model
}

\author{
Marina E. Plissiti and Christophoros Nikou, Senior Member, IEEE
}

\begin{abstract}
A method for the segmentation of overlapping nuclei is presented, which combines local characteristics of the nuclei boundary and $a$ priori knowledge about the expected shape of the nuclei. A deformable model whose behavior is driven by physical principles is trained on images containing a single nuclei, and attributes of the shapes of the nuclei are expressed in terms of modal analysis. Based on the estimated modal distribution and driven by the image characteristics, we develop a framework to detect and describe the unknown nuclei boundaries in images containing two overlapping nuclei. The problem of the estimation of an accurate nucleus boundary in the overlapping areas is successfully addressed with the use of appropriate weight parameters that control the contribution of the image force in the total energy of the deformable model. The proposed method was evaluated using 152 images of conventional Pap smears, each containing two overlapping nuclei. Comparisons with other segmentation methods indicate that our method produces more accurate nuclei boundaries which are closer to the ground truth.
\end{abstract}

Index Terms-Active shape models, microscopic images, modal analysis, overlapping nuclei segmentation, Pap smear images, physically based deformable model, shape priors.

\section{INTRODUCTION}

O NE OF THE most interesting and challenging issues in the automated analysis of microscopic images, is the delineation of the overlapped cells or cells nuclei. The cell overlapping areas in the microscopic slides are very common phenomena, especially in the case of the well-known Pap smear [1]. The Pap smear consists of a sample of cells taken from the cervix and it is extensively used in gynecology as a screening test in order to detect premalignant and malignant processes. With this procedure, the cervical cells are collected from the cervix with a special device and they are then smeared onto a glass slide, which is examined under a microscope in order to identify abnormalities in the structure and morphology of cell nuclei. The segmentation of these images has been studied by several researchers [2]-[9], as the nucleus is the structural part of the cell that presents

Manuscript received November 30, 2011; revised April 7, 2012; accepted June 4, 2012. Date of publication June 26, 2012; date of current version October 12, 2012. This work was supported in part by the European Union (European Regional Development Fund - ERDF) and Greek National Funds through the Operational Program "THESSALY- MAINLAND GREECE AND EPIRUS-2007-2013" of the National Strategic Reference Framework (NSRF 2007-2013). The associate editor coordinating the review of this manuscript and approving it for publication was Prof. Jean-Philippe Thiran.

The authors are with the Department of Computer Science, University of Ioannina, Ioannina 45110, Greece (e-mail: marina@cs.uoi.gr; cnikou@ cs.uoi.gr).

Color versions of one or more of the figures in this paper are available online at http://ieeexplore.ieee.org.

Digital Object Identifier 10.1109/TIP.2012.2206041 significant changes when the cell is affected by a disease. As an example, we can mention that the nuclear border abnormalities are highly correlated with the infection of cells by the Human Pappiloma Virus (HPV) [10] and the shape modifications of the nucleus are associated with the existence of Cervical Intraepithelial Neoplasia (CIN) [11]. Furthermore, features that are based on the nuclei shape have been used for the discrimination of normal and abnormal cells [12]. Thus, the accurate identification of the nucleus shape is important for the correct interpretation of the Pap smear.

Usually, the existence of the different layers of the cervical specimen in the slide results in areas, where the cells of an upper layer partially obscure the cells lying underneath. In real time microscopic examination this problem is commonly solved in most cases with the adjustment of the lens focus, and the cells of different layers are clearly identified. However, in static images acquired through a digital camera adopted on a microscope, this is not possible and for this reason efforts have been made by several researchers in order to contribute to the automated segmentation of the overlapped cells or overlapped nuclei in many cytological images.

More specifically, in terms of the general segmentation techniques used for the separation of overlapped nuclei, the geometric active contours are used in [13]. In this approach, each cell is represented by its own level-set function and a coupling constrain prevents neighboring contours from overlapping each other. Furthermore, the distance transform in a binary image containing the regions of the nuclei is calculated in [14] and the topographic surface generated by the distance transform is considered as a Gaussian mixture. The EM algorithm is then applied for the determination of the parameters of each nucleus cluster. The watershed transform has been extensively used in several studies [15]-[18]. The main concern for these methods is to overcome the oversegmentation and for this purpose, special attention has been paid on the determination of marking strategies and the selection of appropriate nuclei and background markers.

The above methods were applied in different cytological images such as fluorescence in situ hybridization (FISH) images [15], [17] or microscopic images from several specimens such as mammary invasive ductal carcinoma or cervical images [14], [16]. In the first case, the separation line between the clustered nuclei is obtained through the application of the watershed transform. However, these cases are very sensitive to the selection of appropriate markers, in order to identify the correct location of each nucleus marker and a marker for the overlapping area between the nuclei. Furthermore, 
in [13], [16] and [18], the partial nuclei boundaries lying in the areas of overlap are estimated with ellipse fitting algorithms.

In this paper, we present an alternative method for the separation of overlapping nuclei which is based on the representation of the nucleus shape by the vibrations of a springmass system [19] and the statistical learning of the vibration modes of the system in the framework of ASM [20]. More specifically, through physics-based shape parameterization, the elastic 2-D boundary modeling can be achieved by a closed chain topology of virtual masses on the contour. Each node of the model has a mass and it is connected with two neighboring nodes through springs with the same stiffness and damping parameters. The physics-based equations of motion govern the deformation of the model [19], which reaches the equilibrium when it is placed on the object's boundary. Segmentation methods based on these models have been proposed for multimodal brain image analysis [21], skeleton family generator [22] and reconstruction of serially acquired slices for the determination of volumes [23].

In addition, active shape models (ASM) are well-known parametric deformable models which are based on the construction of a statistical model of the global shape variation from a training set of shapes. They have been extensively used for the recognition and localization of objects that follow the same geometric form of a sample of well known shapes, such as face detection [24], biomedical image segmentation [25] and handwritten character recognition [26]. Image segmentation with ASM requires the representation of the shape of the object of interest by a set of points. Based on this representation, a deformable model is iteratively deformed to fit to an instance of the object of interest in an unknown image. The model is constrained by the Point Distribution Model (PDM) [20], in order to vary only in ways that are learnt in a training set of labeled examples.

Our work combines the segmentation of an image with ASM [20] and the representation of an object using modal analysis [19]. Thus, a physical model is adopted in the training phase, in which the parameters to be learnt are the variations of the modes of the model. The attributes of the nuclei shapes are expressed in terms of modal analysis and in the training phase the modal distribution is estimated. Therefore, a more compact description of the shape model is obtained. Next, we develop a framework for the deformation of an active physical model similar to ASM, for the detection of an unknown new nucleus in images containing two overlapped nuclei.

It must be noted that the idea of combining the physicallybased and the active shape models was first proposed in [27]. However, no closed form solution for the deformation of the model was provided, and only admissible deformations in the neighborhood of the initial position of the model were acceptable, providing a heuristically obtained solution for the deformation of the model.

As it will be explained in the following paragraphs, our method significantly differs from the aforementioned work in many perspectives. First of all, we provide a closed form solution for the deformation of the model, which does not depend on trial and error based admissible configurations in the shape of the model, but it is based on the dynamic change of the generalized displacement, in order the model to be attracted from the significant characteristics of the image and also to be consistent with the learnt parameters. Thus, the generalized displacements are updated through gradient based optimization. Furthermore, the model converges to a position close to the desired boundary, as it always provides admissible solutions. This is possible even in the case of the overlapping part of the nuclei where no significant edges are present, as the contribution of the external energy in the model deformation is mutable.

The method proposed herein is motivated by [21]. The main difference is that in [21] the a priori knowledge was obtained for one anatomical structure (the skull) and then, the other structures (e.g. the brain) are estimated by least squares. In our case, the a priori knowledge concerns the occluded parts of the nuclei, whose estimation relies on an iterative gradient descent method resulting naturally from the linear form of the model. Moreover, there are two main differences of our method with respect to standard ASM algorithm: first, the modal amplitudes of the learnt model are used instead of the 2D landmark points and second, the cost function to be minimized for the detection of the nuclei boundaries is affected by the locally adaptive image force, which is introduced in order to extract reliable nuclei boundaries in the regions of overlap. An important characteristic of the proposed method is that it provides a smooth representation of the nucleus boundary by the physical model, which entails in the reduction of the number of parameters employed in the segmentation step. This is possible since the number of the modal amplitudes that contain the most important information about the shape of the model is small, due to the principal components analysis step. Furthermore, another significant characteristic is that the proposed model is flexible and it converges fast in the position of the desired boundary, due to the linear transformation adopted in the deformation step. Finally, the method is evaluated using a test set of 152 cytological images of conventional Pap smears, which contain two overlapped nuclei each and it presents high performance, as it is verified by the results.

\section{Methods}

\section{A. Training Phase}

In this phase, the physical model is constructed and it is applied on a training set of images containing a single nucleus. This is necessary in order to learn the modal distribution describing the shape of the nuclei. For this reason, images containing only a single nucleus are processed, where the entire nucleus border is visible. After the definition of the modal distribution, the models are applied in images containing two overlapping nuclei. The basic steps of the training phase are described in detail in the following paragraphs.

1) Construction of the Physical Model: In order to obtain a compact representation of the shape of the nuclei boundary, we adopt the physical deformable model proposed by Nastar and Ayache [19]. A physics based deformable model is used, whose behaviour is controlled by the governing equations of motion. More specifically, the physical model 
consists of $N$ virtual masses located at points $\mathbf{X}(t)=$ $\left\{\mathbf{x}_{1}(t), \mathbf{x}_{2}(t), \ldots, \mathbf{x}_{N}(t)\right\}$. The motion of the physical model towards the border of the object of interest is expressed by a finite element formulation and is estimated by solving a $2 N$-dimensional differential matrix equation (for the horizontal and vertical direction):

$$
\begin{aligned}
& \mathbf{M} \ddot{\mathbf{U}}_{x}(t)+\mathbf{C} \dot{\mathbf{U}}_{x}(t)+\mathbf{K} \mathbf{U}_{x}(t)=\mathbf{F}_{x}(t) \\
& \mathbf{M U}_{y}(t)+\mathbf{C} \dot{\mathbf{U}}_{y}(t)+\mathbf{K} \mathbf{U}_{y}(t)=\mathbf{F}_{y}(t)
\end{aligned}
$$

where $\mathbf{M}, \mathbf{C}$ and $\mathbf{K}$ are $N \times N$ matrices describing the mass, the damping and the stiffness of the model. Moreover, $\mathbf{F}_{x}$ and $\mathbf{F}_{y}$ are vectors containing the image force at the nodes locations and $\mathbf{U}_{x}, \dot{\mathbf{U}}_{x}, \ddot{\mathbf{U}}_{x}$ and $\mathbf{U}_{y}, \dot{\mathbf{U}}_{y}, \ddot{\mathbf{U}}_{y}$ are the vectors of displacement, velocity and acceleration of the model in the horizontal and vertical direction respectively.

The above equations describe the equilibrium between internal and external forces of the system. The internal forces are expressed by the definition of the virtual masses of the model and the interaction between them, while the external forces are usually defined as the intensity or the gradient of the image at the pixels where the nodes of the model are located. The system (1) can be solved by setting the initial values of displacement and velocity equal to zero and then using an explicit Euler scheme. However, instead of solving directly the equilibrium equation (1), we can use a frequency based technique called modal analysis, which describes the motion of the model in terms of the free vibrations of the system.

More specifically, at a first step the following change of basis is used [21]:

$$
\mathbf{U}=\Phi \tilde{\mathbf{U}}
$$

where $\Phi$ is a square non-singular matrix and $\tilde{\mathbf{U}}$ is the vector of the generalized displacement. The columns of the matrix are selected to be the eigenvectors of the generalized eigenproblem:

$$
\mathbf{K} \phi_{i}=\omega_{i}^{2} \mathbf{M} \phi_{i}
$$

where $\phi_{i}$ is the $i$-th mode and $\omega_{i}$ its frequency. This is an effective way for the expression of the displacement vector $\mathbf{U}$ in terms of modal displacements, that is:

$$
\mathbf{U}=\Phi \tilde{\mathbf{U}}=\sum_{i=1}^{N} \tilde{u}_{i}(t) \phi_{i}
$$

where $\tilde{u}_{i}$ is the amplitude of the $i$-th mode. It can be shown [19] that matrices $\mathbf{K}, \mathbf{M}$ and $\mathbf{C}$ are simultaneously diagonalized by

$$
\begin{aligned}
& \Phi^{T} \mathbf{M} \Phi=\mathbf{I} \\
& \Phi^{T} \mathbf{K} \Phi=\Omega^{2}
\end{aligned}
$$

where $\mathbf{I}$ is the identity matrix and $\Omega^{2}$ is the diagonal matrix whose elements are the eigenvalues $\omega_{i}, i=1, \ldots, N$.

Premultiplying (1) by $\Phi^{T}$ and substituting the displacement vector with its equivalent form in (2) leads to:

$$
\ddot{\tilde{\mathbf{U}}}+\tilde{\mathbf{C}} \dot{\tilde{\mathbf{U}}}+\tilde{\Omega}^{2} \tilde{\mathbf{U}}=\tilde{\mathbf{F}}
$$

where $\tilde{\mathbf{C}}=\Phi^{T} \mathbf{C} \Phi$ and $\tilde{\mathbf{F}}=\Phi^{T} \mathbf{F}$. The above matrix-form equation can be decoupled for each dimension into $N$ scalar equations of the form:

$$
\ddot{\tilde{u}}_{i}(t)+\tilde{c}_{i} \dot{\tilde{u}}_{i}(t)+\tilde{\omega}_{i}^{2} \tilde{u}_{i}(t)=\tilde{f}_{i}(t) .
$$

The solution of these equations at time $t$ leads to the calculation of the amplitudes $\tilde{u}_{i}(t), i=1, \ldots, N$ and the deformation of the model is estimated using the modal superposition equation (4). At each time step, the new positions of the nodes of the model $\mathbf{X}(t)$ are given by

$$
\mathbf{X}(t)=\mathbf{X}\left(t_{0}\right)+\mathbf{U}(t)
$$

where $\mathbf{X}\left(t_{0}\right)$ is the vector containing the initial spatial positions of the model and $\mathbf{U}(t)$ is the nodal displacement vector.

In practice, the nodal displacements $\mathbf{U}(t)$ are approximated by $\hat{\mathbf{U}}(t)$ using a fraction of the modes of vibration, which present the highest amplitudes, that is:

$$
\hat{\mathbf{U}}(t)=\sum_{i=1}^{l} \tilde{u}_{i}(t) \phi_{i}
$$

where $l \ll N$. For the choice of the number of modes $l$, the total energy is calculated by:

$$
E=\sum_{i=1}^{N} \tilde{u}_{i}^{2}
$$

and we chose the first $l$ amplitudes carrying a predefined percentage of the total energy.

An issue that must be clarified is the calculation of the eigenvectors and eigenvalues of the generalized problem of (3). From the classical theory of vibration of a crystal lattice, it can be proved that the relationship between spatial $(k)$ and temporal $(\omega)$ frequencies is given by:

$$
\omega^{2}(p)=\frac{4 K}{M} \sin ^{2}\left(\frac{k(p) \alpha}{2}\right) .
$$

In (11), due to the periodicity of the closed chain:

$$
k(p) \alpha=\frac{2 \pi p}{N}, \quad p \in \mathcal{B}(\mathcal{N})
$$

where $\mathcal{B}(\mathcal{N})$ is the Brillouin zone [19]:

$$
\mathcal{B}(\mathcal{N})= \begin{cases}{\left[-\frac{N}{2}+1, \ldots, \frac{N}{2}\right],} & \text { for } N \text { even } \\ {\left[-\frac{N-1}{2}, \ldots, \frac{N-1}{2}\right],} & \text { for } N \text { odd }\end{cases}
$$

Combining (11) and (12) we can calculate the temporal frequencies $\omega_{i}^{2}$, which correspond to the eigenvalues of the problem in (3). The corresponding eigenvectors $\phi(p)$ are then given by:

$$
\phi(p)=\left[\ldots, \cos \frac{2 \pi p}{N}, \ldots\right]^{T} .
$$

Thus, using (14), analytic forms for the eigenvectors are obtained and the motion of the model can be easily expressed in terms of frequency modes as described in (9). 
2) Training the Physical Model: Instead of describing the object of interest by a set of $N$ labeled landmark points as in the standard ASM algorithm, we focus on the learning of the generalized displacements $\tilde{\mathbf{U}}$ of the model, in the images of the training set, which contains a single nucleus each. This is an equivalent representation, since the combination of (2) and (8) results in the spatial coordinates of the shape.

In the training phase, the nuclei boundaries were manually traced by an expert in all the images of the training set. An issue that must be taken into account for the correct training of the model is that the shapes in each image must be registered. The boundaries of the nuclei are commonly smooth and they do not present any specific characteristic point that can be considered as landmark point, in order to use it for the registration of the shapes. Thus, we are restricted to use the characteristics of the elliptical appearance of the boundaries for the registration of the shapes. The obtained shapes of the nuclei are ellipsoidal, having a major and a minor axis, and these characteristics are used for the registration of the shapes. In our work, we register all the obtained shapes automatically, in such a way that their major axis is placed on the same horizontal line and their center of gravity coincide. Based on this registered boundary, the distance transform was estimated for every image. On the resulted image, a physical model was initialized and deformed until convergence, in order to detect the desired boundary. As a result, an accurate nucleus boundary was obtained.

From the final shape of the model, the generalized displacement vector $\tilde{\mathbf{U}}$ was estimated and from the entire training set, the mean $\overline{\tilde{\mathbf{U}}}$ was calculated, which entails in the representation of the mean shape of the nucleus boundary. Furthermore, the covariance matrix $\mathbf{S}$ of the vectors was also calculated. Using principal component analysis (PCA), the eigenvectors $\mathbf{a}_{i}$ of the covariance matrix $\mathbf{S}$ are used for an equivalent representation of the shape, that is

$$
\tilde{\mathbf{U}}=\overline{\tilde{\mathbf{U}}}+\mathbf{A b}
$$

where $\mathbf{A}$ is the matrix with columns the eigenvectors $\mathbf{a}_{i}$ and b is a vector containing the model coordinates in the basis of the eigenvectors:

$$
\mathbf{b}=\mathbf{A}^{T}(\tilde{\mathbf{U}}-\overline{\tilde{\mathbf{U}}}) \text {. }
$$

Taking into account the $J$ eigenvectors which correspond to the $J$ largest eigenvalues of the covariance matrix, the shape can be approximated by:

$$
\tilde{\mathbf{U}} \simeq \overline{\tilde{\mathbf{U}}}+\mathbf{A}_{J} \mathbf{b}_{J}=\overline{\tilde{\mathbf{U}}}+\left[\begin{array}{llll}
\mathbf{a}_{1} & \mathbf{a}_{2} & \cdots & \mathbf{a}_{J}
\end{array}\right]\left[\begin{array}{llll}
b_{1} & b_{2} & \cdots & b_{J}
\end{array}\right]^{T}
$$

where $\mathbf{A}_{J}$ and $\mathbf{b}_{J}$ are derived from $\mathbf{A}$ and $\mathbf{b}$ by using only the $J$ selected eigenvectors. Thus, using the mean vector $\overline{\tilde{\mathbf{U}}}$ and (9), the mean shape of the nuclei is described and this will be used as an initial template, in order to separate the overlapped nuclei in the images of the test set.

\section{B. Segmentation of Overlapping Nuclei Boundaries}

This procedure includes the determination of the initial positions of the two models in the image and the deformation process that the models follow until convergence. A graphical

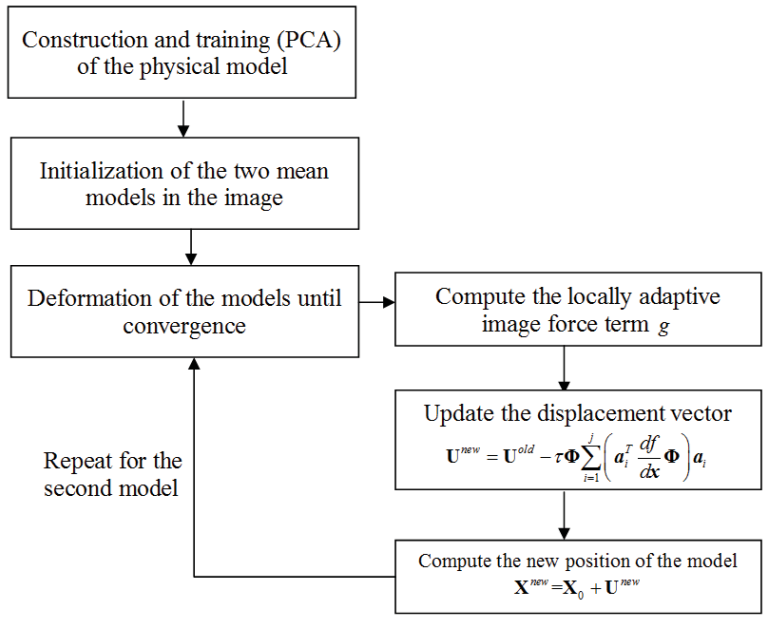

Fig. 1. Basic steps of the segmentation of the overlapping nuclei (see text for details).

description of this step is depicted in Fig. 1 and the details are described in the following paragraphs.

1) Initialization of the Model: The most important prerequisite of our method in order to provide reliable results is the accurate localization of the initial model. If the initial model is not close to the real boundary, then the results would probably be highly erroneous, as the model would converge in local minima of the image, which do not correspond to the real nucleus boundary. The mean nuclei boundary that has been determined in the training phase stands as the initial model of the nucleus boundary. As we are looking for two nuclei in the images of the test set, the initial positions of two models must be detected close to the real nuclei boundaries. For this reason, each image is first preprocessed for the detection of the strong nuclei edges, which will force the mean nuclei boundary to be located near to the real one through chamfer matching [28].

More specifically, in the grayscale counterpart of the initial image, we first reduce the noise by applying a Gaussian filter. Then, using histogram equalization technique, the contrast of the nuclei and the background is enhanced. In order to avoid the inhomogeneities inside the nuclei areas, which are commonly present due to uneven staining of the smear, we proceed with the formation of homogenous minima intensity valleys. This is feasible with the application of the H-minima transform in the original image [29]. Thus a marker image is constructed by the subtraction of a threshold value $h$ from every pixel of the complement of the initial image. Then through the grayscale reconstruction process, we obtain an image that contains the regional minima, whose depth is less than $h$, suppressed. The result of this process is depicted in Fig. 2(b), where a rough description of the positions of the two overlapped nuclei is defined. In this image, the Canny edge detector is applied (Fig. 2(c)), and some strong boundary edges are detected. The distance transform $g$ is then calculated.

In the resulting image we search for the best two matching positions of the initial model (Fig. 2(d)). The measure of correspondence between the edges and the model is the sum of the pixel values at which the model is located. A perfect 


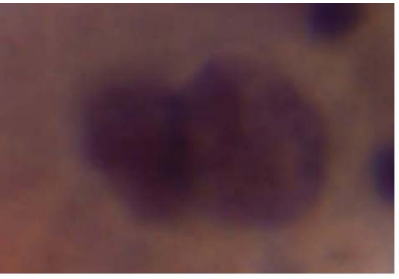

(a)

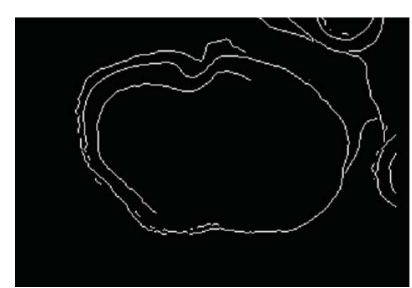

(c)

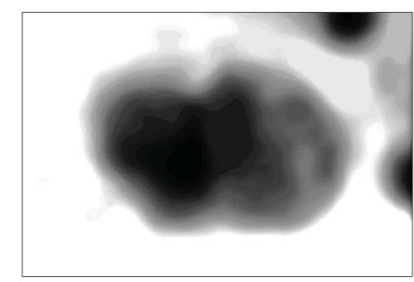

(b)

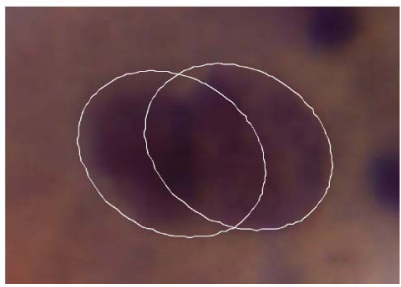

(d)
Fig. 2. Initialization of the two models. (a) Initial image. (b) Image obtained after the noise reduction, histogram equalization, and $\mathrm{H}$-minima transform of the grayscale counterpart of the initial image. (c) Result of the application of the Canny edge detector in (b). (d) Initial placement of the learnt model on each nucleus, after the chamfer matching. This figure is best viewed in color.

match would produce a zero value in this measure (as the model would perfectly match in the edges of the image, in which the value of the distance transform is zero). However, as this is an extremely rare case in real images, we search for the position of the model in the image that minimizes this sum.

It must be noted that in each image the nuclei size and orientation may vary. Thus, the initial model is rotated by a step of $1^{\circ}$ angle and scaled by factors between 0.6 and 1.2 of its original position and size. In this way, more accurate initial approximations of the nuclei boundaries are detected. After the detection of the initial position of the models, we proceed with the deformation of the models in order to converge to the final nuclei boundaries.

2) Deformation of the Models: Using the shape representation defined in (17), the algorithm fits the desired model in the image, driven by the image characteristics and the prior training. In each iteration, the changes in the generalized displacements should be consistent with the learnt parameters, and this is feasible by the minimization of a cost function $f(\tilde{\mathbf{U}})=g\left(\mathbf{X}_{0}+\Phi \tilde{\mathbf{U}}\right)$, where $g$ is the distance transform of the image as it is defined in the initialization step and its argument is the deformed shape with respect to (8) by omitting the temporal dependency for simplicity. More specifically, in each step, the algorithm selects the new generalized displacements by the following optimization schemes:

$$
\min _{\tilde{\mathbf{U}}} f(\tilde{\mathbf{U}})=\min _{b_{i}, i=1, \ldots, J} f\left(\overline{\tilde{\mathbf{U}}}+\left[\mathbf{a}_{1} \mathbf{a}_{2} \cdots \mathbf{a}_{J}\right]\left[b_{1} b_{2} \cdots b_{J}\right]^{T}\right) .
$$

The gradient descent scheme of (18) with the new variables $b_{i}$ is given by:

$$
b_{i}^{\text {new }}=b_{i}^{\text {old }}-\tau\left(\mathbf{a}_{i}^{T} \frac{d f}{d \tilde{\mathbf{U}}}\right)
$$

where $\tau$ is the time step and:

$$
\frac{d f}{d \tilde{\mathbf{U}}}=\frac{d f}{d \mathbf{X}} \frac{d \mathbf{X}}{d \tilde{\mathbf{U}}}=\frac{d f}{d \mathbf{X}} \Phi .
$$

The term $\frac{d f}{d \mathbf{X}}=\left[\nabla g\left(x_{1}, y_{1}\right), \ldots, \nabla g\left(x_{N}, y_{N}\right)\right]^{T}$ is actually the gradient of the image force term, where $g$ is again the distance transform of the image as it is described above. In terms of the original variable $\tilde{\mathbf{U}}$, the update rule (19) turns out to be

$$
\begin{aligned}
\tilde{\mathbf{U}}^{\text {new }} & =\tilde{\mathbf{U}}^{\text {old }}-\tau \sum_{i=1}^{J}\left(\mathbf{a}_{i}^{T} \frac{d f}{d \tilde{\mathbf{U}}}\right) \mathbf{a}_{i} \\
& =\tilde{\mathbf{U}}^{\text {old }}-\tau \sum_{i=1}^{J}\left(\mathbf{a}_{i}^{T} \frac{d f}{d \mathbf{X}} \Phi\right) \mathbf{a}_{i} .
\end{aligned}
$$

Notice that the initial value for $\tilde{\mathbf{U}}$ is the mean shape obtained with the training of ASM. Premultiplying (21) by $\Phi$ to the left we get

$$
\Phi \tilde{\mathbf{U}}^{\text {new }}=\Phi \tilde{\mathbf{U}}^{\text {old }}-\tau \Phi \sum_{i=1}^{J}\left(\mathbf{a}_{i}^{T} \frac{d f}{d \mathbf{X}} \Phi\right) \mathbf{a}_{i}
$$

Regarding (19), we have

$$
\mathbf{U}^{\text {new }}=\mathbf{U}^{\text {old }}-\tau \Phi \sum_{i=1}^{J}\left(\mathbf{a}_{i}^{T} \frac{d f}{d \mathbf{X}} \Phi\right) \mathbf{a}_{i} .
$$

Finally, from (8), the local positions of the landmark points are calculated by:

$$
\mathbf{X}^{\text {new }}=\mathbf{X}_{0}+\mathbf{U}^{\text {new }}
$$

where $\mathbf{X}_{0}$ is the initial position of the model in the image.

For the determination of a stopping criterion for the iterative procedure of the deformation of the models, we observe their shape in two sequential steps, and we consider that they converge when no significant change is noticed. For this reason, we calculate the Euclidean distance between the points of the previous and the current state of the model. If the difference in the Euclidean distance is smaller than a very small value (in our experiments smaller than $10^{-3}$ ) then the deformation of the models is stopped. This parameter is fixed for every image of our data set.

3) Spatially Adaptive Image Force: The image force is defined as the force due to the potential field created by the image characteristics. The most common approach is to use the image gradient magnitude as the external force, in order to guide the deformable model in the areas of the image where high gradients are located (which usually imply the existence of strong edges of the objects of interest). However, the limitation of the gradient image force becomes evident in parts of the image with smooth intensity transitions, in which the gradient magnitude is very low. In Pap smear images, the staining procedure introduces variances in illumination and dye concentration. In some cases the nuclei borders may not be clearly distinguishable from the background, and these locations present weak image gradient. If a deformable model is initialized in such locations, it is not probable to guide it toward an edge. Thus, the gradient-based force field has a 
limited capture range for the deformable model. The distance potential force alleviates this issue for binary images.

As we can observe from (23) and (24), the deformation of the model is also controlled by the image force term. The degree of the influence of the image force term in the motion of the deformable model can be modulated by setting appropriate weight values $w_{1}, w_{2}, \ldots, w_{n}$ at each image point that belongs to the model, and the image force can be defined as $\frac{d f}{d \mathbf{X}}=$ $\left[w_{1} \nabla g\left(x_{1}, y_{1}\right), \ldots, w_{N} \nabla g\left(x_{N}, y_{N}\right)\right]^{T}$.

Thus, if the weights of this term have large values, the model will deform mainly according to the image characteristics. On the other hand, if the image force weight is small, the model deformation would be driven by the learnt nuclei shape. In images of overlapping nuclei, the edges of the isolated part of the nuclei boundary must be taken into account, in order to attract the model for the detection of the true nuclei boundaries. In those points, high value of the image force term is desirable. However, in the area of overlap, there is no edge information and with the use of high weight values, the model could be attracted from the boundary of the isolated part of the neighbor nucleus, resulting in a high erroneous identification of the real nucleus boundary. An immoderate example of the influence of the values of the image force weights in the deformation of the models is depicted in Fig. 3, where we attempt to emphasize the influence of different weight values in the final segmentation results. For this reason, we have used extreme weight values and we let the deformable model move without any restrictions, using (22) and (23). As we can observe, the segmentation results are highly erroneous.

In order to avoid such phenomena, we use different values for the weights in the image force term, depending on the position of the point in the boundary. Thus, in each step of the deformation of the two models, the area of overlap is determined, and the points of the models lying in this area are associated with small weight values compared with the weight values in the non overlapping area, as it is explained in the next paragraphs. Therefore, the influence of the image force in the deformation of this part of the model is limited. However, in the rest of the points of the model, we use large values for the image term weights, and this results in the detection of the actual nuclei boundaries in the non-overlapping area of each nucleus. The PCA coordinates $\mathbf{a}_{i}$ estimated by the nonoverlapping part of the model yield a set of modal amplitudes which determine the behavior of the rest of the points based on the learnt vibrations.

\section{EXPERIMENTAL RESULTS AND DISCUSSION}

\section{A. Study Group}

In our experiments, for the construction of the training set we have selected 300 images, which are used for the estimation of the modal distribution of the physical model in the training phase. Each of these images in the training set, contains a single normal nucleus of conventional Pap smear.

Furthermore, the proposed method was tested in terms of the accurate determination of the nuclei boundary on a test set of 152 images containing two overlapping nuclei each, yielding 304 nuclei in total. Thus, the training and the test set of images

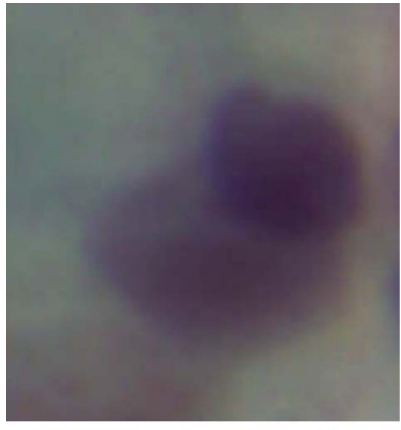

(a)

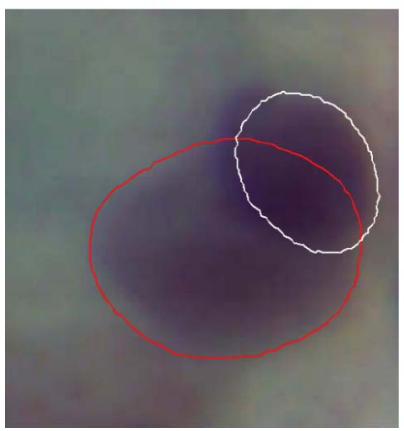

(c)

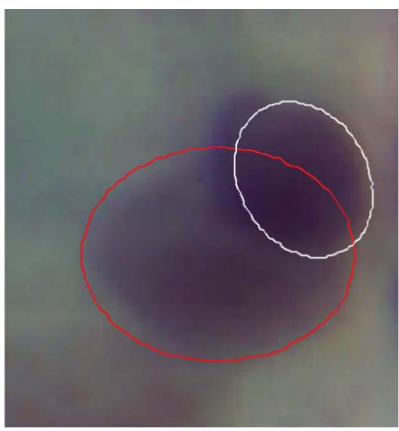

(b)

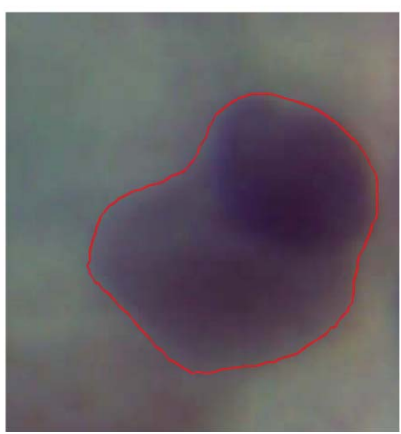

(d)
Fig. 3. (a) Initial image. (b) Initial position of the two models. Results obtained using (c) small and (d) large weight values for the image force of one of the two nuclei. Notice that in (c), there exist small differences from the initial position of the models. In (d), however, the model of one nucleus converges in a position of high gradient of the image, resulting in the erroneous identification of the boundary of the area containing both of the nuclei. This figure is best viewed in color.

are independent. All of these images were acquired through a CCD camera (Olympus DP71) adapted to an optical microscope (Olympus BX51). We have used a $40 \times$ magnification lens and the acquired images were stored in JPEG format. The initial images obtained by the optical microscope have size $3072 \times 4080$ pixels. These images are then cropped manually in order to construct the database of images containing the overlapped nuclei and a small portion of the neighboring background (such as in Fig. 2, 3, 6 and 7). The average size of all the images in our database is $273 \times 315$ pixels.

\section{B. Numerical Evaluation}

For the evaluation of the performance of the method, the boundaries of the nuclei in the entire test set were estimated manually, after careful examination of an expert cytopathologist. The determination of the boundary of each nucleus in the overlapping area was based on the exploitation of small variances in the intensity of this area, which an expert could identify. However, in many cases, there was no existence of intensity variances, and the boundary of each nucleus was manually drawn by the expert, following the expected shape of the nucleus in the specific image. Based on the ground truth, we have calculated the area of overlap in our data set, and the overlapping area varies between $4.0 \%$ and $48.2 \%$ with $20.03 \pm 11.32(\%)($ mean \pm std).

In order to evaluate the performance of the proposed method, the Euclidean and Hausdorff distance of the final 
position of the model and the ground truth was calculated in each image. The presented method underwent a twofold evaluation: a) we have tested the influence of the use of different weight values in the final segmentation compared to the use of one single value and b) the results of the method were compared to the results of different segmentation methods, such as the standard ASM algorithm using different weight values in the area of overlap (as it is proposed in our method), the conventional technique of the ellipse fitting in the overlapped nuclei boundary incorporated in our method (instead of using different image force weight values), the unsupervised segmentation of overlapped nuclei using Bayesian classification [14] and the H-minima transformbased marker extraction and contour parameterization method for segmenting overlapped nuclei [16]. It must be noted that the ellipse fitting technique is extensively used from several researchers ([14], [16], [18]), in order to estimate the nucleus boundary in the overlapping areas and it is based on the hypothesis that the nuclei shape is generally ellipse-like. Thus, in each step of the deformation of the model, the points of the boundary of the nucleus of the non-overlapping area are used for the calculation of the interpolated ellipse using the direct least squares fitting of ellipses [30]. Then, the part of the boundary in the overlapping area is completed using the corresponding part of the interpolated ellipse and the link points are smoothed (they are calculated as the average of the previous and the next point of the model).

The choice of the weights in our work is based on a two step procedure, which is performed in a portion of images of the test set (50 images), and the selected weight values are used for the entire set of the images containing the overlapped nuclei. More specifically, at first, we use the same value for the weights of overlapping and non-overlapping areas, and we test the method for several different values for the weights. The Hausdorff distance between the result of the proposed method and the ground truth using a single value for the image force term is depicted in Fig. 4. As we can see, the best results were obtained for $w_{1, \ldots, n}=0.1$ and the Hausdorff distance for this weight is 24.29. However, with the use of different weight values, the corresponding Hausdorff distance is reduced, which indicates that the use of different weight values renders the method more performing. Thus, in the next step, having found that the best segmentation was obtained with the weight value 0.1 (Fig. 4), we keep this value constant for the external weights in non overlapping areas and we test multiple values for the weights in the overlapping areas. This is described in Fig. 5, which contains the segmentation results of the method from several experiments, where we have used different weight values for the overlapping areas, while the weight value in non overlapping areas was fixed to 0.1 . From this image, we can observe that the best segmentation results were obtained for $w_{1, \ldots, k}=0.001$, where $k$ is the number of the points of the model lying in the overlapping areas.

The comparative results of the proposed method and the other segmentation techniques are included in Table I. As we can see, our method exhibits better performance, since both the Euclidean and Hausdoff distances are smaller compared with the other methods, and it does not exhibit large vari-

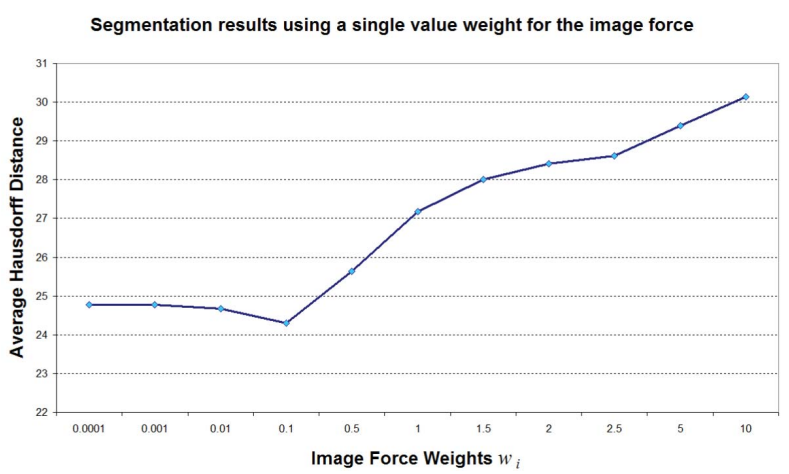

Fig. 4. Result of the proposed method using the same value for the image force term, for all points of the model. Notice that best results are obtained using a value of 0.1 and the corresponding Hausdorff distance is 24.29.

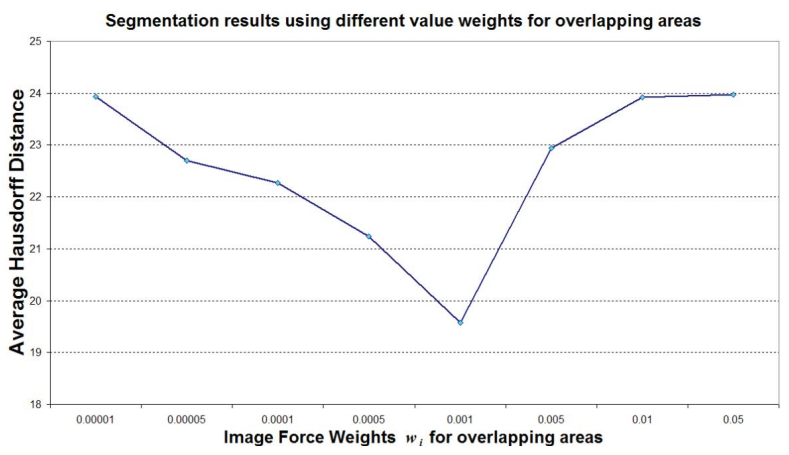

Fig. 5. Result of the proposed method using the same value $w_{i}=0.1$ for the image force term in the points lying in the nonoverlapping area of the nuclei, and multiple values $w_{1,2}, \ldots, k$ for the $k$ points of the model lying in the overlapping area. Notice that best results are obtained using a value of 0.001 and the corresponding Hausdorff distance is 19.58 .

TABLE I

Results of Overlapped NuClei Segmentation Methods in Terms of Euclidean ANd Hausdorff Distances (MEAN \pm STD)

\begin{tabular}{lll}
\hline \hline & Hausdorff & Euclidean \\
\hline Proposed method & $19.91 \pm 8.38$ & $8.71 \pm 3.45$ \\
Proposed method with ellipse fitting & $20.88 \pm 10.14$ & $8.77 \pm 3.82$ \\
ASM & $21.76 \pm 11.30$ & $9.91 \pm 4.01$ \\
H-minima [16] & $22.98 \pm 11.76$ & $10.96 \pm 4.30$ \\
Bayesian classification [14] & $24.59 \pm 12.44$ & $11.13 \pm 4.50$ \\
\hline
\end{tabular}

ations, as the standard deviation is small. This implies that the proposed model is closer to the manually traced nuclei boundaries, and as a result it is more accurate than the other approaches. Furthermore, based on the experimental results and the overlapping percentage of our image data set (as it was described in the above paragraphs), we can conclude that our method can successfully segment nuclei that present grade of overlap lower than $50 \%$.

Several examples of the segmentation results of our method are depicted in Fig. 6. The segmentation results in images of Fig. 7(a) of our method and several methods proposed in the literature are depicted in Fig. 8. As we can see, the use of the ellipse fitting algorithm for the overlapped parts of the nuclei in our method produces some rugged points in 

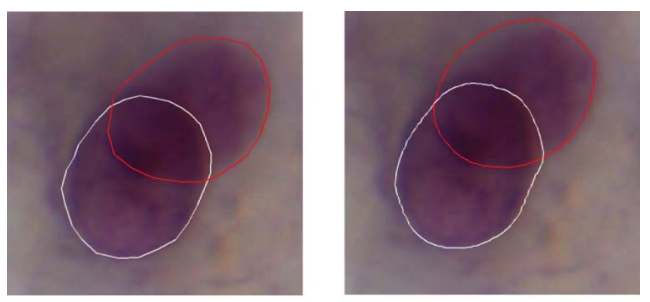

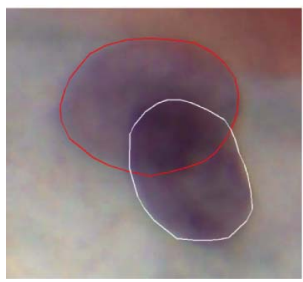

(a)

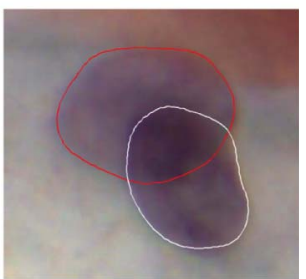

(b)
Fig. 6. Segmentation results for the proposed method. (a) Ground truth and (b) segmentation. This figure is best viewed in color. (a)

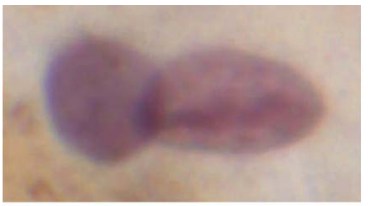

(b)

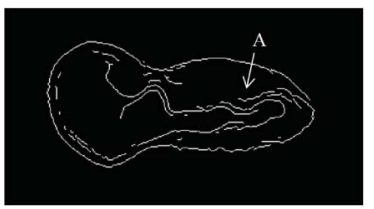

(c)

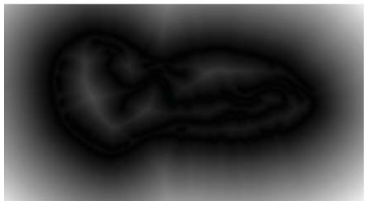

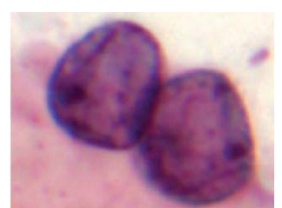
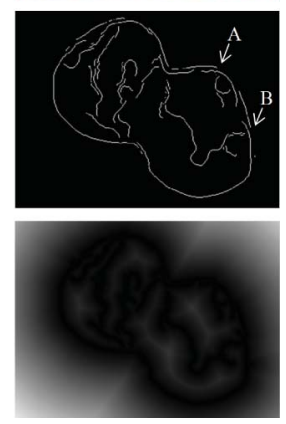

Fig. 7. (a) Representative images containing overlapped nuclei. (b) Edge images resulted after the application of the Canny edge detector (initialization step). The point $\mathrm{A}$ in the first image indicates some falsely detected edges in the inner area of the nucleus. The points A and B in the second image indicate the existence of discontinuities. (c) Corresponding distance transform.

the nuclei boundaries, and the boundaries of the overlapping areas are not well detected, as they do not follow an elliptical form in the overlapping areas. Furthermore, the standard ASM algorithm produces noisy results and this indicates that the representation of the shape of the nuclei by the vibrations of a spring-mass system instead of landmark points, assures a smooth representation of the shape, and as a result more accurate boundaries for the nuclei.

It must be noted that in our work, we have trained the ASM having as training shapes the nuclei boundaries obtained with the convergence of the physical model in the training set, and not with independently distributed points as it is the standard procedure. By these means, the ASM method is unbiased with respect to the manual tracing of the nuclei and the comparison of the two methods may be performed on the same basis. Besides, these shapes can be considered as an accurate representation of the nuclei boundaries, since the mean Euclidean distance of the final position of the physical (a)

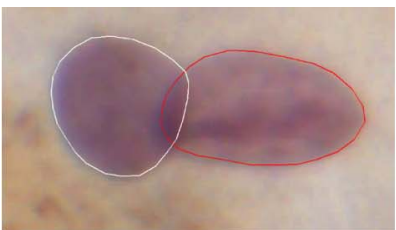

(b)

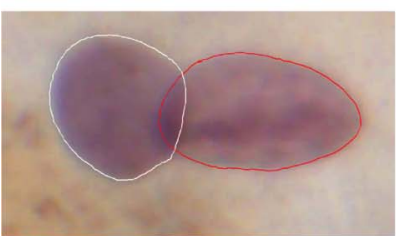

(c)

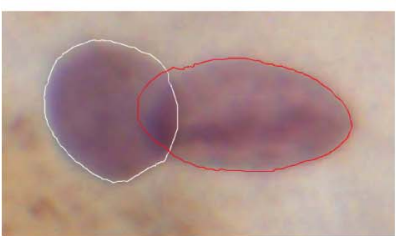

(d)

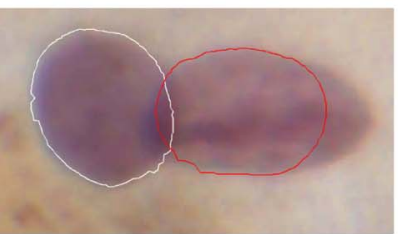

(e)

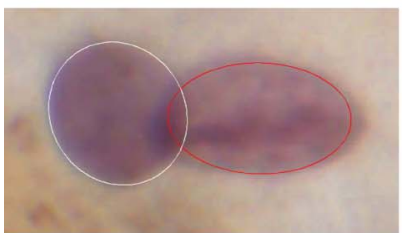

(f)
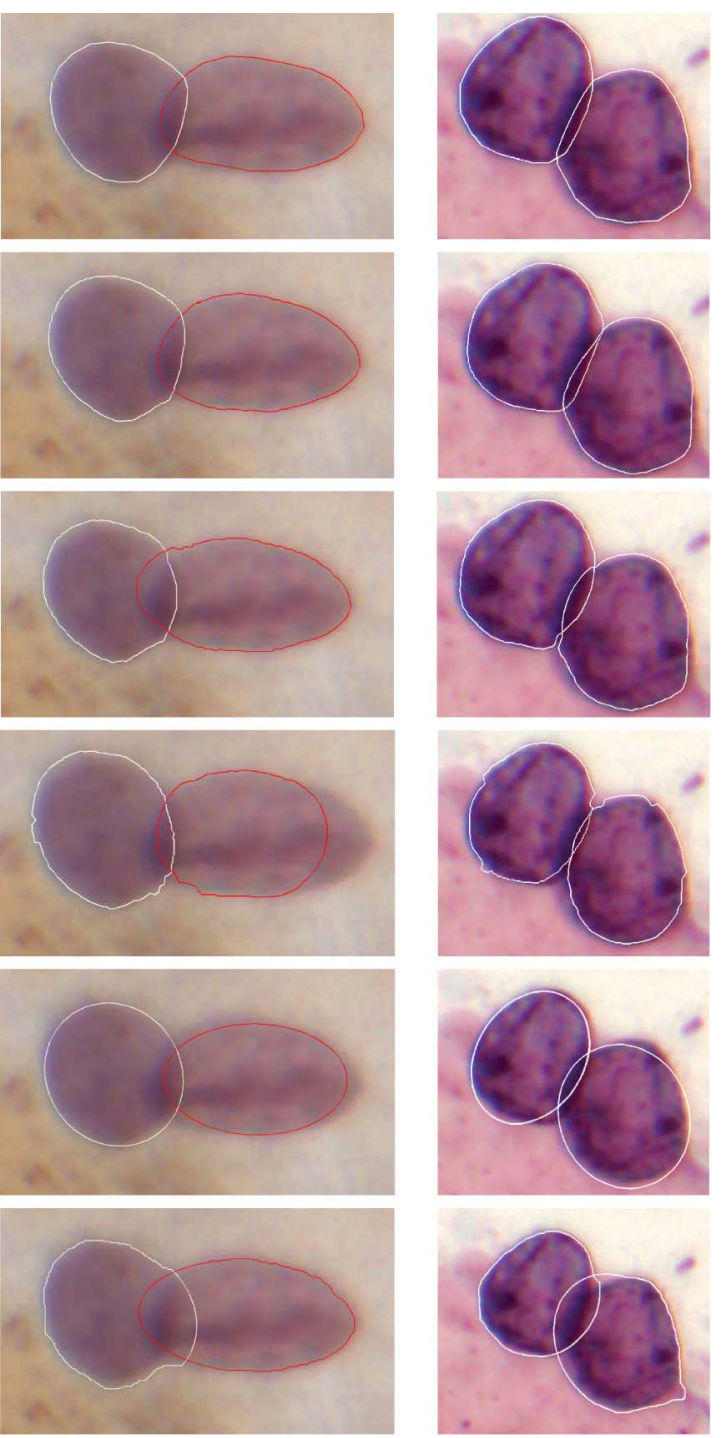

Fig. 8. Segmentation results. (a) Ground truth in the initial image. The segmentation results for (b) proposed method, (c) ellipse fitting algorithm, (d) standard ASM algorithm, (e) H-minima marker extraction [16], and (f) Bayesian [14]. Notice that in (c), elliptical parts produced by the ellipse fitting algorithm for the nucleus boundary in the overlapping areas miss the true boundary of the nuclei, as it does not follow the specific elliptical distribution in these areas. Furthermore, the nuclei boundary presents some noisy parts as the transition from the overlapping to the nonoverlapping part of the boundary is not smooth. Moreover, the results produced by the standard ASM algorithm in (d) are highly erroneous. In (e), ellipses produced by the $\mathrm{H}$-minima marker extraction method proposed in [16] are rough approximations of the true nucleus boundary. Finally, in (f), nuclei boundaries marked with white present rugged points. This figure is best viewed in color.

model and the ground truth is 1.68 pixels in the images of the training set. For this reason, the main eigenvectors for the ASM model do not exhibit significant differences from the main eigenvectors of the physical model. However, the noisy results of the ASM method are due to the minimization procedure, in which the ASM is more sensitive to the image force. This can be observed in Fig. 8(d), where the discontinuities in the detected edges [Fig. 7(b), second image, points A and B] produce rugged segments of points in the convergence of ASM. Furthermore, the detected edges in the inner area of the nucleus [Fig. 7(b), first image, point A] seem to interfere 
to the convergence of the model, which fails to identify the actual nuclei borders. The reason for this effect is that in the standard ASM algorithm, the image force term $\frac{d f}{d \mathbf{X}}$ has a direct influence on the new variables (the projection of the points of the model on the eigenvector space), which are then multiplied by the eigenvectors to obtain the new position of the model. On the other hand, in our case, the new variables (the projection of the generalized displacements on the eigenvector space) change according to $\frac{d f}{d \tilde{\mathbf{U}}}$ in (19), or equivalently according to $\frac{d f}{d \mathbf{X}} \Phi$ in (23). Thus, the new position of the model depends on the specific product, which provides a smooth image force term. In this way, the model is not affected by the potential noise contained in the image force.

For the methods proposed in [14] and [16] the results are not quite accurate, and this is a consequence of several reasons. In [16] the outcome is actually the ellipse obtained by the detected nuclei boundary points produced by adaptive thresholding. As we can observe in Fig. 8(e), the estimated ellipses provide a rough approximation of the actual nuclei boundaries and additional processing is required for the detection of more refined nuclei borders. The method in [16] may be considered similar to the initialization step of our algorithm; however, there are several differences with our method. More specifically, in the initialization step, we use the H-minima transform for the construction of smooth intensity valleys and the reduction of the noise in the image, in contrast to [16], in which the H-minima transform is used in a marker extraction scheme. For this reason we have used only one threshold value for $h$ (Table II) and we do not test many threshold values as it is proposed in [16]. Furthermore, the edges of the image are detected with the Canny edge detector, in order to obtain a binary image, without the use of global thresholding or the watershed transform as in [16]. In the binary image we try all possible similarity transformations, in order to achieve a reliable initial position of our model, which is known through the training step. By the time we locate the model in its initial position, we continue with the minimization process and the gradient descent method is independent of these parameters.

Furthermore, in [14] the boundaries of the non-overlapping area of the nuclei are obtained after morphological operations and adaptive thresholding. Although this procedure is fast, it does not always succeed in the detection of accurate boundaries. As we can see in Fig. 8 (f, first image, white traced nucleus), the nucleus boundary that was erroneously detected in the bottom of the non-overlapping area, results in the estimation of an ellipse for the overlapping area that does not correspond to the expected nuclei boundaries. Furthermore, in Fig. 8 (f, second image, white traced nucleus) we can observe that the inhomogeneity in the image intensity results in the wrong detection of the nucleus boundary in the nonoverlapping area (near the bottom, right hand corner of the image). Therefore, we may conclude that in images with noise and artifacts (like Pap smear images), adaptive thresholding techniques do not provide accurate detection even for the nonoverlapping boundaries of the nuclei and further processing is required.

Moreover, as a measure of the computational efficiency of the segmentation method, we present in Table III the mean
TABLE II

PARAMETER VALUES USED IN THE EXPERIMENTS

\begin{tabular}{lr}
\hline \hline Parameter & Value \\
\hline$h$ (H-minima transform) & 20 \\
Stiffness $K$ & 5 \\
Mass $M$ & 1 \\
Damping $\tilde{c}_{i}, i=1, \ldots, N$ & 1 \\
Radius of the initial model & 85 \\
Model points & 120 \\
Initial time step $\tau_{0}$ & 0.001 \\
Weights of image force (overlapping area) & 0.001 \\
Weights of image force (non overlapping area) & 0.1 \\
Number of modes $\tilde{u}_{i}$ & 14 \\
Number of eigenvectors $\mathbf{a}_{i}$ & 4 \\
\hline
\end{tabular}

TABLE III

EXECUTION TIME (MEAN \pm STD)

\begin{tabular}{lr}
\hline \hline Segmentation method & Time $(\mathrm{sec})$ \\
\hline Proposed method & $17.73 \pm 0.82$ \\
Proposed method with ellipse fitting & $45.37 \pm 3.39$ \\
Standard ASM & $27.01 \pm 2.65$ \\
H-minima [16] & $2.94 \pm 0.69$ \\
Bayesian classification [14] & $4.62 \pm 1.32$ \\
\hline
\end{tabular}

times for the processing of each image (including the initialization and the deformation of the two models), developed in Matlab using a Pentium $2.0 \mathrm{GHz}$ with $3 \mathrm{~GB}$ RAM. As we can observe, the computational burden that the ellipse fitting algorithm introduces in each step of the deformation of the model, results in an increase of the processing time of each image. Furthermore, the reduction of the learnt parameters, as it is described in (17), renders our method superior to the standard ASM algorithm. In the cases of non iterative procedures, in which an ellipse fitting algorithm is implemented such as in [14] and [16] the required processing times are clearly shorter than in the rest of the methods. In both of these methods, the estimation of a suitable ellipse that fits the points in the non overlapping area is calculated once, and no iteration is performed. In contrast, the computational cost of the ellipse fitting algorithm in our method is bigger, because the ellipse is calculated in every iteration of the algorithm. The iterative procedure that we propose for the convergence of the model, although it requires more computational time, is necessary for the refinement of the nuclei borders in both the overlapping and non-overlapping areas, which is not achieved by the non-iterative methods. This can be easily verified by the comparative results in Table I, where we can observe that the non-iterative methods have lower performance than the proposed method.

The parameters of the steps of the method were selected after several tests (Table II). Thus, the threshold $h$ in the $\mathrm{H}$-minima transform in the initialization of the models is set to 20. For the calculation of modal vibrations in (11), we selected $K=5$ and $M=1$. Furthermore, in (7), the value for all $\tilde{c}_{i}, i=1, \ldots, N$ was set to 1 . For the initialization of the physical model, a circle of radius 85 pixels and 120 points 
in its circumference was used centered at the centroid of the registered manually traced shape.

It must be noted that the change of the weight values for a large number of points in a single step may lead to a large displacement of the deformable model, as the additive value of the image force term in (23) would be large. In our implementation we have eliminated this effect with a variable time step, which is calculated in each iteration. Thus, given an initial time step $\tau_{0}=0.001$, the time step in (23) is calculated as $\tau_{t}=\tau_{0}(1-O P R)$, where $O P R$ is the overlapping points ratio, which is defined as the percentage of the points of the physical model lying in the overlapping area. By these means, a weighted sum of the contribution of the points in the nonoverlapping and in the overlapping area is obtained which ensures the stability of the algorithm, as the deformation of the model is smooth and it avoids abrupt changes.

From (9), we have calculated that the first 14 modes $\tilde{u}_{i}$ contain more than $99 \%$ of the total energy in each image of the training set. Thus, only 14 parameters (instead of 120 landmark points) are sufficient for the accurate representation of the desired shape. Furthermore, after the application of PCA in these learnt parameters, only 4 eigenvectors which correspond to the highest eigenvalues lead to an almost exact shape representation, as they represent the $99.9 \%$ of the total energy. This clarifies that the proposed segmentation method provides a more compact shape representation which results in the reduction of the parameters to be learnt.

\section{Relation Between the Weight Values and the Overlapping Percentage}

In order to investigate the influence of the selected weight values compared to the degree of overlapping, we have performed an experiment on a synthetic image, constructed from two individual images of the training set, each one containing a single nucleus. In these images, we have selected the area of each nucleus based on the ground truth. Then, in a new image, the areas of the nuclei were incrementally overlapped, and we have compared the segmentation results of our method with respect to the degree of overlapping of the nucleus area and the selected value weights. To this end, we have calculated the Hausdorff distance of the obtained boundary of one nucleus of interest, whose area overlapping was ranging from $10 \%$ to $90 \%$ (Fig. 9). The gray level value of the area of overlap of the examined nucleus is set to $95 \%$ of the initial gray level. The rest of the nuclei areas contained the same intensity value as in the original images and the background was set to the mean value of both images. It must be noted that, in the synthetic image, we considered that the initialization step produced acceptable initial positions for the models.

The method was applied in this image for the drawing of conclusions about the influence of different rates between the weight values of overlapping and non overlapping areas, and how they affect the segmentation results with respect to the degree of overlap. Thus, the weight value for the points lying in the non-overlapping area was fixed to 0.1 for all the images. Then, the method was applied using different values for the weights of the nodes belonging to the overlapping areas. These weights were set to 2, 5, 10, 20, 100, 200 and

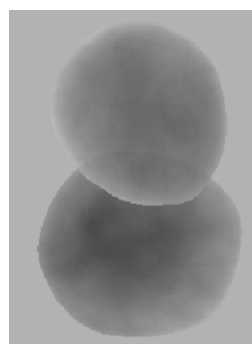

(a)

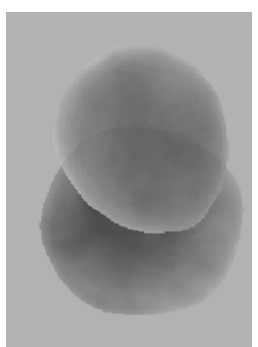

(b)

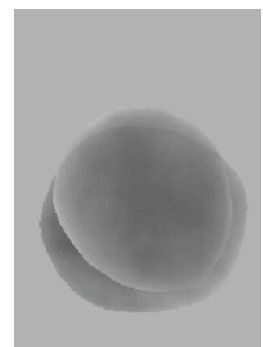

(c)
Fig. 9. Construction of a synthetic image. The area overlapped by the upper nucleus is (a) $10 \%$, (b) $50 \%$, and (c) $90 \%$.

1000 times lower than the value of the weights of the nodes in the non overlapping areas. Furthermore an experiment with equal weight values of the overlapping and non-overlapping areas (set to 0.1) was also performed. The degree of overlap of the nucleus of interest in the synthetic images varied from $10 \%$ to $90 \%$. As we can see from Fig. 10, the choice of equal value for the weights produces worse results for almost all the cases of overlapping. Furthermore, values lower than 100 times of the non-overlapping weight value have the same result in the Hausdorff distance. In general, we can conclude that for overlapping percentage greater than $45 \%$ the best results were obtained using a weight value for the overlapping areas which is half than the weight value for the non overlapping areas. For smaller overlapping rates, the best results are obtained using a weight value of overlapping areas which is at least five times smaller than the weight value for the non overlapping areas. It must be noted, that the Hausdorff distance for this experiment is generally small, as the image of overlapping nuclei is, in a way, ideal, since it does not contain any noise or background artifacts.

\section{Robustness to Noise}

The synthetic images described above were also used for the evaluation of the performance of our method in the presence of noise. Since the convergence of the models depends on the existence of edges, we created an inhomogeneous background in the synthetic images, in order to present edges that are due to noise rather than the existence of a nucleus border. For this reason, in the Canny edge detector we have tested the values of $\{0.001,0.05,0.10,0.15,0.20,0.25,0.30\}$ for the maximum threshold, and the values for the minimum threshold are automatically computed as 0.4 times lower than the maximum threshold. The corresponding signal-to-noise ratios for these thresholds are $\{-20.50,-18.59,-14.1,-9.39,-5.25,-2.05$, $-0.27\}$ in $\mathrm{dB}$. It must be noted that we define the signalto-noise ratio as the number of pixels of the ground truth boundary to the number of pixels of the spurious edges. An example of this experiment in an image presenting 30\% of overlapping degree is depicted in Fig. 11.

Furthermore, in order to quantify the edge pixels that are close to the real boundaries and have significant influence to the deformation of the model, we have also calculated an index which expresses the sum of the distance of noise edges from the ground truth and it is given as $D_{e d g e s}=\sum 1 / d_{i}^{2}$, 


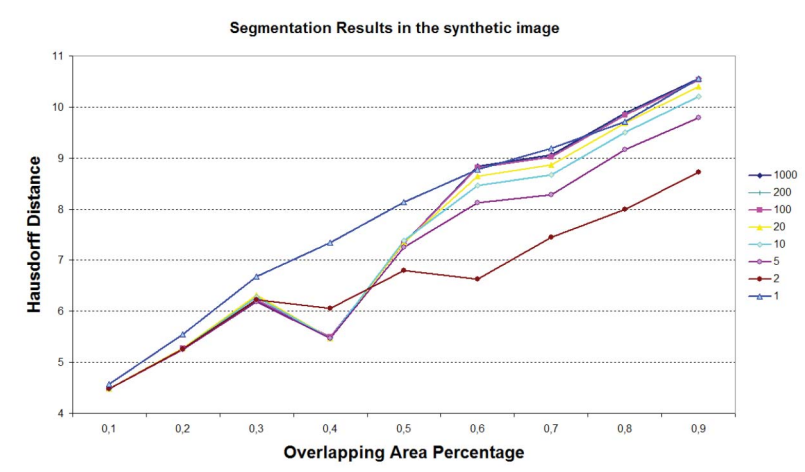

Fig. 10. Hausdorff distance in the synthetic image with respect to the nuclei overlapping percentage. The curves correspond to the segmentation obtained using the specific rates of the weight values of the nonoverlapping areas to the weight values of the overlapping areas. (a)

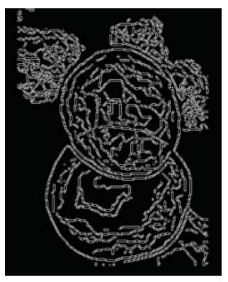

(b)

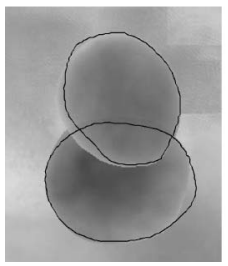

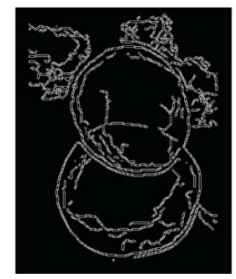

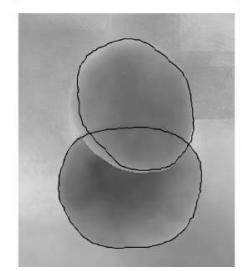

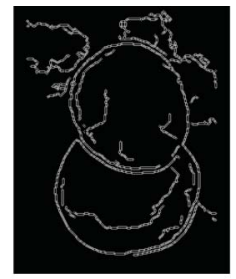

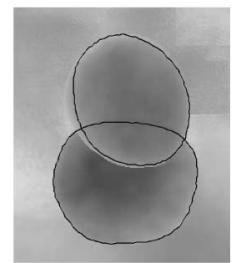

Fig. 11. (a) Edge images obtained with the use of different threshold values in the Canny edge detector. (b) Corresponding results after the application of our method.

where $d_{i}$ is the smallest distance of the pixel from the ground truth boundary. Thus, edge pixels that are near to the ground truth have larger contribution to this index. In Fig. 12, we can observe that this index grows disproportionately to the SNR, demonstrating that a large number of noise edges are close to the ground truth in high levels of noise.

For the evaluation of the performance of the method for each amount of noise, the Hausdorff distance was calculated and the results in terms of the mean and the standard deviation for all the overlapping degrees with respect to the signal-to-noise ratio are depicted in Fig. 13. As we can see, the maximum value of Hausdorff distance is 10.17 and corresponds to SNR $=-20.5$, which indicates that the method produces acceptable results, even in the presence of severe noise.

\section{E. Robustness to Initial Condition}

As it was already mentioned, a critical condition for the accurate definition of the nuclei boundaries through the convergence of the two models, is the initialization of the models close to the area of the real boundaries. In our method, this is accomplished with an exhaustive search described in section II-B.1, which aims at the detection of the most probable positions of the two rotated and scaled mean models in the image. In order to investigate the influence of a different

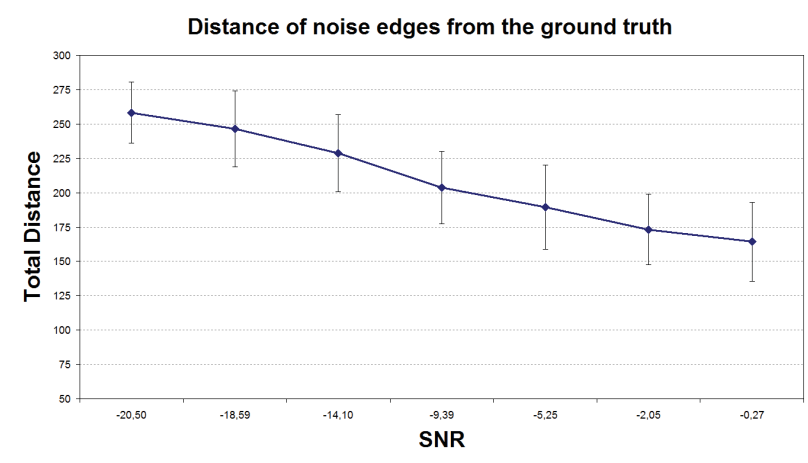

Fig. 12. Total distance of the edge pixels from the ground truth in terms of the mean and the standard deviation for all of the overlapping degrees with respect to the signal-to-noise ratio.

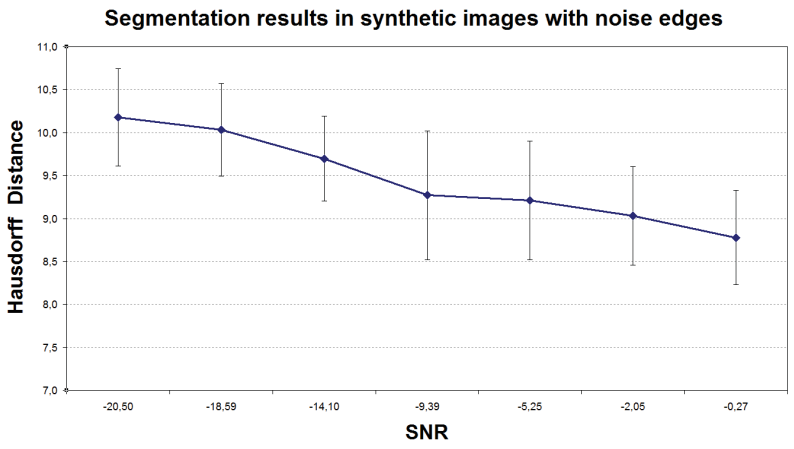

Fig. 13. Segmentation results in terms of the mean and the standard deviation of the Hausdorff distance for all of the overlapping degrees with respect to the signal-to-noise ratio.

initial position of the models, we have used a method proposed by Thévenaz et al. called "the ovuscule" [31] in all of the images of our database. This method results in the definition of two ellipses in the image, which are considered as initial approximations of the boundaries of the nuclei.

More specifically, in the grayscale counterpart of the initial image, we performed global thresholding [32] and computed the distance transform. Then, the ovuscules were initialized as circles centered at the two maxima of the image, which were separated by a distance of at least 73 pixels, as this is the mean radius of the nuclei in the training set (Fig. 14(a)). After the optimization process, the resulted ovuscules (Fig. 14(b)) were considered as the initial models in the image. As we can see in Fig. 15, the initialization of the models differs both in orientation and size for the two nuclei. The proposed method was applied in a second step. The Hausdorff and Euclidean distance of the obtained nuclei boundaries with this configuration were calculated as $19.98 \pm 8.36$ and $8.75 \pm 3.46$ respectively.

Regarding Table I, we can conclude that the segmentation results in terms of the ground truth are approximately similar. This implies that the proposed method is robust to different initializations, given that the two models are initialized in an area sufficiently close to the ground truth. On the other hand, the processing time required in this experiment was measured $10.11 \pm 0.63 \mathrm{sec}$, and from Table III it is clear that the use of the ovuscules has a remarkable positive effect on the required processing time of the method. This is an expected result as 


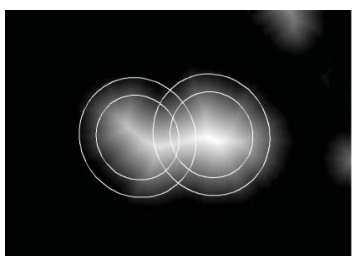

(a)

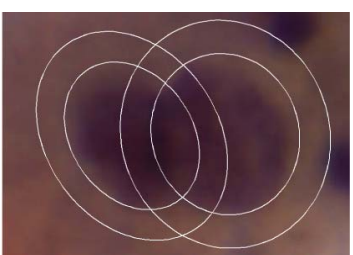

(b)
Fig. 14. (a) Initialization of the two ovuscules [31] in the image obtained after global thresholding and the computation of the distance transform. (b) Ellipses obtained by the ovuscules. The inner ellipses are considered as the initial positions of the models of our method.

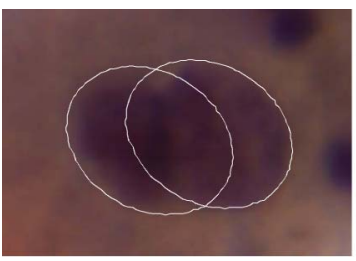

(a)

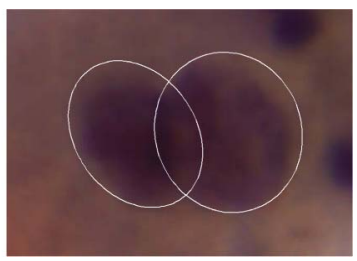

(b)
Fig. 15. Different initializations of the two models using (a) proposed method and (b) ovuscules.

the time for the initialization of the models is reduced, since we avoid the exhaustive search, which is time demanding, and the ovuscules converge to their final position very fast.

\section{F. Experiments in Images With Several Number of Nuclei}

Our method can identify the boundary of a single nucleus in an image, and also it can be extended to be able to segment three or more overlapped nuclei. In the case of one nucleus, we initialize a single model, and then we let the model deform with all its nodes having the same weight value, as there is no overlapping. We have applied the method in 200 images containing a single nucleus. It must be noted that these images were not used for the training. The performance of the method in terms of the Hausdorff distance was $10.13 \pm 2.31$ pixels, which implies that the method can successfully detect the boundaries of single nuclei with high precision. Representative results are depicted in Fig. 16.

Furthermore, we performed several experiments in images containing three and four overlapping nuclei. The main steps for the application of the method remain the same as the models deform independently. The only differences with respect to two nuclei are (a) the initialization of the models in the image and (b) the detection of the overlapping area. In the first case, the process which is described in section II.B.1 could be followed, but instead of two models, we search for the suitable positions of a number of models equal to the number of the nuclei in the image. In the second case, for each model, the area of overlap could be defined as the union of the areas of all the models. Thus, the weights of the specific model would be defined accordingly. Following these steps, we tested our method in 11 images containing three overlapped nuclei and the Haussdorf distance was $15.62 \pm 4.31$. The same experiment was performed in 5 images of four overlapping nuclei and the corresponding distance was $18.19 \pm 3.58$.

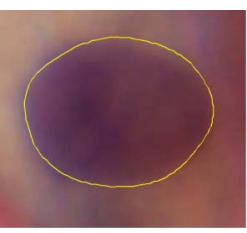

(a)

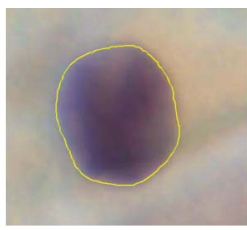

(b)

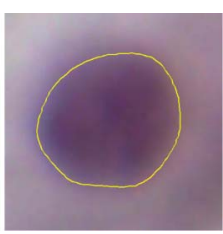

(c)
Fig. 16. (a)-(c) Results of the proposed method for the identification of the boundary of a single nucleus in an image.

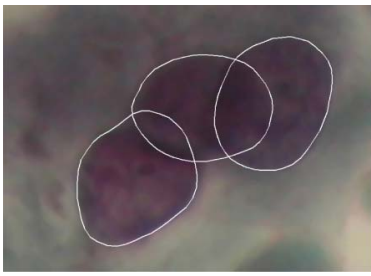

(a)

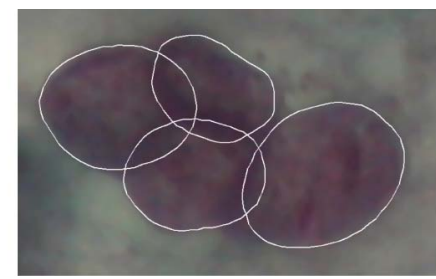

(b)
Fig. 17. Results of the proposed method in images containing (a) three and (b) four overlapping nuclei.

Several examples of the performance of our method in these images are depicted in Fig. 17

As it is verified by the results, the method is able to recognize the correct nuclei boundaries and presents high performance with regard to Hausdorff distance from the ground truth. However, as the number of the images of multiple nuclei that the method was applied is limited, we consider these results as promising though preliminary, and we intent to select a larger number of multiple nuclei images in order to estimate the general behavior of our method.

\section{CONCLUSION}

We have developed a segmentation method combining the physically based model, which provides a compact representation of the shape of the object of interest, and the active shape model, which takes advantage of the a-priori knowledge of the expected shape. The introduction of variable weights in the contribution of the image force in the deformation of the model results in the correct identification of the part of the nuclei boundary that lies in the overlapping areas. The method has been tested in terms of the accurate segmentation of the nuclei borders in images from Pap smear slides, and as it was verified by the results it presents a high performance. Thus, the method produces more accurate nuclei boundaries which are closer to the ground truth, compared to the standard ASM algorithm and the segmentation obtained by two methods proposed for the segmentation of overlapped nuclei. The main advantage of the proposed method is that it provides a flexible way for the simultaneous recognition of the isolated and the overlapped nucleus boundary. This avoids the development of an additional algorithm for the detection of the nuclei boundaries in occluded areas, such as the ellipse fitting algorithm. The proposed method in its current form is automated when the number of the nuclei is known in the images. In the case where the images contain unknown number of nuclei, then an initial step of counting the existed nuclei is necessary, as in [16]. 
As a future work, the method could be easily extended to an automated cell image segmentation system for the analysis of images obtained directly from an optical microscope. This is feasible since the automated detection of the nuclei positions in such images has been successfully addressed in [8] and [9], and the automated construction of subimages similar to those in our test set is possible. Finally, we intend to examine in a more extended data set the efficiency of our method in images containing three or more overlapped nuclei.

\section{ACKNOWLEDGMENT}

The authors would like to thank Dr. A. Charchanti for her valuable help.

\section{REFERENCES}

[1] G. N. Papanicolaou, "A new procedure for staining vaginal smears," Science, vol. 95, no. 2469, pp. 438-439, 1942.

[2] P. Bamford and B. Lovell, "Unsupervised cell nucleus segmentation with active contours," Signal Process., vol. 71, no. 2, pp. 203-213, 1998.

[3] Z. Li and K. Najarian, "Biomedical image segmentation based on shape stability," in Proc. 14th IEEE Int. Conf. Image Process., Sep.-Oct. 2007, pp. 281-284.

[4] M. H. Tsai, Y. K. Chan, Z. Z. Lin, S. F. Yang-Mao, and P. C. Huang, "Nucleus and cytoplast contour detector of cervical smear image," Pattern Recognit. Lett., vol. 29, no. 9, pp. 1441-1453, 2008.

[5] S. F. Yang-Mao, Y. K. Chan, and Y. P. Chu, "Edge enhancement nucleus and cytoplast contour detector of cervical smear images," IEEE Trans. Syst., Man, Cybern. B, Cybern., vol. 38, no. 2, pp. 353-366, Apr. 2008.

[6] C. H. Lin, Y. K. Chan, and C. C. Chen, "Detection and segmentation of cervical cell cytoplasm and nucleus," Int. J. Imag., Syst. Technol., vol. 19, no. 3, pp. 260-270, 2009.

[7] C. W. Chang, M. Y. Lin, H. J. Harn, Y. C. Harn, C. H. Chen, K. H. Tsai, and C. H. Hwang, "Automatic segmentation of abnormal cell nuclei from microscopic image analysis for cervical cancer screening," in Proc. 3rd IEEE Int. Conf. Nano-Molecular Med. Eng., Oct. 2009, pp. 77-80.

[8] M. E. Plissiti, C. Nikou, and A. Charchanti, "Automated detection of cell nuclei in pap smear images using morphological reconstruction and clustering," IEEE Trans. Inf. Technol. Biomed., vol. 15, no. 2, pp. 233241, Mar. 2011.

[9] M. E. Plissiti, C. Nikou, and A. Charchanti, "Combining shape, texture and intensity features for cell nuclei extraction in Pap smear images," Pattern Recognit. Lett., vol. 32, no. 6, pp. 838-853, 2011.

[10] J. Monsonego, Emerging Numbers on HPV Infections: From Science to Practice. Basle, Switzerland: Karger, 2006.

[11] E. Artacho-Perula, R. Roldan-Villalobos, J. Salas-Molina, and R. Vaamonde-Lemos, "Histomorphometry of normal and abnormal cervical samples," Analyt. Quantitat. Cytol. Histol., vol. 15, no. 4, pp. 290-297, 1993.

[12] Y. Marinakis, M. Marinaki, and G. Dounias, "Particle swarm optimizaton for Pap-smear diagnosis," Expert Syst. Appl., vol. 35, no. 4, pp. 1645 1656, 2008.

[13] C. Zimmer and J. C. Olivo-Marin, "Coupled parametric active contours," IEEE Trans. Pattern Anal. Mach. Intell., vol. 27, no. 11, pp. 1838-1842, Nov. 2005.

[14] C. Jung, C. Kim, S. W. Chae, and S. Oh, "Unsupervised segmentation of overlapped nuclei using Bayesian classification," IEEE Trans. Biomed. Eng., vol. 55, no. 12, pp. 2825-2832, Dec. 2010.

[15] J. Cheng and J. C. Rajapakse, "Segmentation of clustered nuclei with shape markers and marking function," IEEE Trans. Biomed. Eng., vol. 56, no. 3, pp. 741-748, Mar. 2009.

[16] C. Jung and C. Kim, "Segmenting clustered nuclei using H-minima transform-based marker extraction and contour parameterization," IEEE Trans. Biomed. Eng., vol. 57, no. 10, pp. 2600-2604, Oct. 2010.

[17] F. Cloppet and A. Boucher, "Segmentation of complex nucleus configurations in biological images," Pattern Recognit. Lett., vol. 31, no. 8, pp. 755-761, 2010.

[18] X. Bai, C. Sun, and F. Zhou, "Splitting touching cells based on concave points and ellipse fitting," Pattern Recognit., vol. 42, no. 11, pp. 2434 2446, 2009
[19] C. Nastar and N. Ayache, "Frequency-based nonrigid motion analysis: Application to four dimensional medical images," IEEE Trans. Pattern Anal. Mach. Intell., vol. 18, no. 11, pp. 1067-1079, Nov. 1996.

[20] T. F. Cootes, C. J. Taylor, and J. Graham, "Active shape models-their training and application," Comput. Vis. Image Understand., vol. 1, no. 1, pp. 38-59, 1995.

[21] C. Nikou, G. Bueno, F. Heitz, and J. P. Armspach, "A joint physics-based statistical deformable model for multimodal brain image analysis," IEEE Trans. Med. Imag., vol. 20, no. 10, pp. 1026-1037, Oct. 2001.

[22] S. Krinidis and V. Chatzis, "A skeleton family generator via physicsbased deformable models," IEEE Trans. Image Process., vol. 18, no. 1, pp. 1-11, Jan. 2009.

[23] S. Krinidis, C. Nikou, and I. Pitas, "Reconstruction of serially acquired slices using physics-based modeling," IEEE Trans. Inf. Technol. Biomed., vol. 7, no. 4, pp. 394-403, Dec. 2003.

[24] K. W. Wan, K. M. Lan, and K. C. Ng, "An accurate active shape model for facial feature extraction," Pattern Recognit. Lett., vol. 26, no. 15, pp. 2409-2423, 2005

[25] B. van Ginneken, A. F. Frangi, J. J. Staal, B. M. ter H. Romeny, and M. A. Viergever, "Active shape model segmentation with optimal features," IEEE Trans. Med. Imag., vol. 21, no. 8, pp. 924-933, Aug. 2002.

[26] D. Shi, S. R. Gunn, and R. I. Damper, "Handwritten Chinese radical recognition using nonlinear active shape models," IEEE Trans. Pattern Anal. Mach. Intell., vol. 25, no. 2, pp. 277-280, Feb. 2003.

[27] A. Garrido and N. P. de la Blanca, "Physically-based active shape models: Initialization and optimization," Pattern Recognit., vol. 31, no. 8, pp. 1003-1017, 1998

[28] G. Borgefors, "Hierarchical chamfer matching: A parametric edge matching algorithm," IEEE Trans. Pattern Anal. Mach. Intell., vol. 10, no. 6, pp. 849-865, Nov. 1988.

[29] P. Soille, Morphological Image Analysis: Principles and Applications. New York: Springer-Verlag, 1999.

[30] A. W. Fitzgibbon, M. Pilu, and R. B. Fischer, "Direct least square fitting of ellipses," IEEE Trans. Pattern Anal. Mach. Intell., vol. 21, no. 5, pp. 476-480, May 1999.

[31] P. Thévenaz, R. Delgado-Gonzalo, and M. Unser, "The ovuscule," IEEE Trans. Pattern Anal. Mach. Intell., vol. 33, no. 2, pp. 382-393, Feb. 2011.

[32] N. Otsu, "A threshold selection method from gray-level histograms," IEEE Trans. Syst., Man, Cybern., vol. 9, no. 1, pp. 62-66, Jan. 1979.

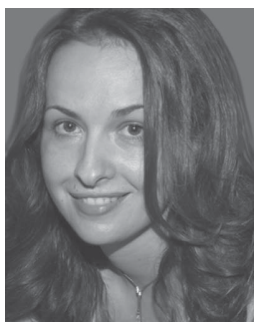

Marina E. Plissiti received the B.Sc., M.Sc., and Ph.D. degrees in computer science from the Department of Computer Science, University of Ioannina, Ioannina, Greece, in 1998, 2001, and 2012, respectively.

She has been involved in research on the processing of biomedical images, such as X-RAY image processing, intravascular ultrasound (IVUS) image segmentation, 3-D reconstruction of coronary arteries through IVUS images and angiography, and microscopic cervical cell image analyses and classification. Her current research interests include medical image processing and artificial intelligence in biomedical applications.

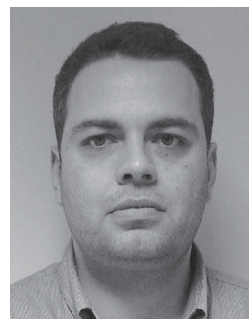

Christophoros Nikou (S'97-M'05-SM'11) received the Diploma degree in electrical engineering from the Aristotle University of Thessaloniki, Thessaloniki, Greece, in 1994, and the D.E.A. and Ph.D. degrees in image processing and computer vision from Louis Pasteur University, Strasbourg, France, in 1995 and 1999, respectively.

He was a Senior Researcher with the Department of Informatics, Aristotle University of Thessaloniki in 2001. From 2002 to 2004, he was a Research Engineer and Project Manager with Compucon S.A., Thessaloniki. He was a Lecturer with the Department of Computer Science, University of Ioannina, Ioannina, Greece, from 2004 to 2009, where he has been an Assistant Professor, since 2009. His current research interests include image processing and computer vision and their application to medical imaging.

Dr. Nikou is an Associate Editor for the EURASIP Journal on Advances in Signal Processing. He is a member of the EURASIP. 